Molecules 2003, 8, 342-349

molecules

ISSN 1420-3049

http://www.mdpi.org/

\title{
Synthesis of New Cyano-Substituted bis-Benzothiazolyl Arylfurans and Arylthiophenes
}

\author{
Livio Racanè ${ }^{1}$, Vesna Tralić-Kulenović ${ }^{1}$ David W. Boykin ${ }^{2}$ and Grace Karminski-Zamola ${ }^{3, *}$ \\ ${ }^{1}$ Faculty of Textile Technology, University of Zagreb, Pierottijeva 6, 10000 Zagreb, Croatia \\ 2 Department of Chemistry and Center for Biotechnology and Drug Design, Georgia State University, \\ Atlanta, Georgia 30303-3083, USA \\ 3 Department of Organic Chemistry, Faculty of Chemical Engineering and Technology, University of \\ Zagreb, Marulicev trg 20, 10000 Zagreb, Croatia
}

*Author to whom the correspondence should be addressed; e-mail:gzamola@pierre.fkit.hr

Received: 12 December 2002; in revised form: 11 March 2003 / Accepted: 27 March 2003 /

Published: 31 March 2003

\begin{abstract}
The new compounds 2-[4-(6-cyanobenzothiazol-2-yl)phenyl]-5-(6-cyanobenzothiazol-2-yl)furan (6a) and 2-[4-(6-Cyanobenzothiazol-2-yl)phenyl]-5-(6-cyanobenzothiazol-2-yl)thiophene $(\mathbf{6 b})$ were synthesized by multi-step reactions from the corresponding 2-furan and 2-thiophene carboxaldehydes (route A), as well as from 2furan and 2- thiophene carboxylic acids (route B). Route B involves one less step than route $\mathrm{A}$, but the overall yields of the reactions are considerably lower.
\end{abstract}

Keywords: bis-Benzothiazole, 6-Cyanobenzothiazole, Furan, Thiophene.

\section{Introduction}

Benzothiazoles are heterocyclic compounds with multiple applications and, although they have been known from long ago to be biologically active, [1,2,3], their varied biological features are still of great scientific interest nowadays. They show, for example, very intensive antitumor activity, especially the phenyl-substituted benzothiazoles [4-6], while condensed pyrimido[2,1-b]benzothiazoles 
and benzothiazolo[2,3-b]-quinazolines exert antiviral activity [7]. Recently, Racanè at al. [8] have described the synthesis of bis-substituted amidinobenzothiazoles as potential anti-HIV agents. Substituted benzamido- and phenylacetamido-substituted 2-phenylbenzothiazoles [9,10,11], 2substituted 6-nitro- and 6-aminobenzothiazoles [12], fluorobenzothiazoles [13] and Schiff bases derived from benzothiazoles [14] show microbiological activity.

On the other hand, bis-benzothiazoles and substituted bis-benzothiazoles are frequently fluorescent compounds and therefore convenient for fluorimetric measurements, which could serve as a potential method for detection of binding the biologically active compounds on DNA [15]. However, there is little data describing compounds containing two benzothiazole rings attached via a heterocyclic system, such as 2,5-benzothiazolylfuran and thiophene and its derivatives [16,17,18], as well as their vinylogues $[19,20]$.

\section{Results and Discussion}

Cyano substituted bis-benzothiazolyl compounds $\mathbf{6 a}$ and $\mathbf{6 b}$ were synthesized starting from 2furan- and 2-thiophenecarboxaldehydes or from 2-furan- and 2-thiophenecarboxylic acids via multistep reactions by the two routes designated $\mathrm{A}$ and $\mathrm{B}$, respectively (Scheme 1).

In the first step of the reaction, 2-furan- or 2-thiophenecarboxaldehydes, and 2-furan- or 2thiophenecarboxylic acids were arylated by the diazonium salts of $p$-aminobenzoic acid by the well known Meerwein arylation procedure [20,21]. In agreement with the greater aromaticity of thiophenes with respect to furans, it is evident from the arylation yields that the furan derivatives are more reactive than the corresponding thiophene derivatives. Thus, 2-furancarboxaldehyde yields $60 \%$ of 1a [20] upon arylation; 2-furancarboxylic acid yields $58 \%$ of $\mathbf{2 a}$ [20], 2-thiophenealdehyde yields $17 \%$ of $\mathbf{1 b}$ [21] and 2-thiophenecarboxylic acid yields $19 \%$ of $\mathbf{2 b}$, respectively.

Benzothiazolyl compounds 3a and 3b, synthesized from the appropriate aldehydes and 4-amino-3mercaptobenzonitrile [22], according to a modified condensation method [18,23], have been converted to the corresponding chlorocarbonyl derivatives $\mathbf{4 a}$ and $\mathbf{4 b}$. In the last step (route A), these chlorocarbonyl compounds were condensed with 4-amino-3mercaptobenzonitrile to obtain the biscyanobenzothiazolyl compounds $\mathbf{6 a}$ and $\mathbf{6 b}$ in good yields of about $75 \%$.

The syntheses of compounds $\mathbf{6 a}$ and $\mathbf{6 b}$ (route B) have been carried out with 5-(4-carboxyphenyl)2-furylcarboxylic acid (2a) [20] and 5-(4-carboxyphenyl)-2-thienylcarboxylic acid (2b). Dicarboxylic compounds $\mathbf{2 a}$ and $\mathbf{2} \mathbf{b}$ have been converted to the dichlorocarbonyl derivatives $\mathbf{5 a}$ and $\mathbf{5 b}$ and then these were condensed with 4-amino-3-mercaptobenzonitrile in yields of about 35\%. Route B has one step less then route A, but the overall yield of the reactions is considearbly lower.

\section{Acknowledgments}

This study was financially supported by the Ministry of Science of the Republic of Croatia within project No. 125005, and US-Croatian Scientific and Technogical Cooperation Award No. JF 146. 


\section{Scheme 1}

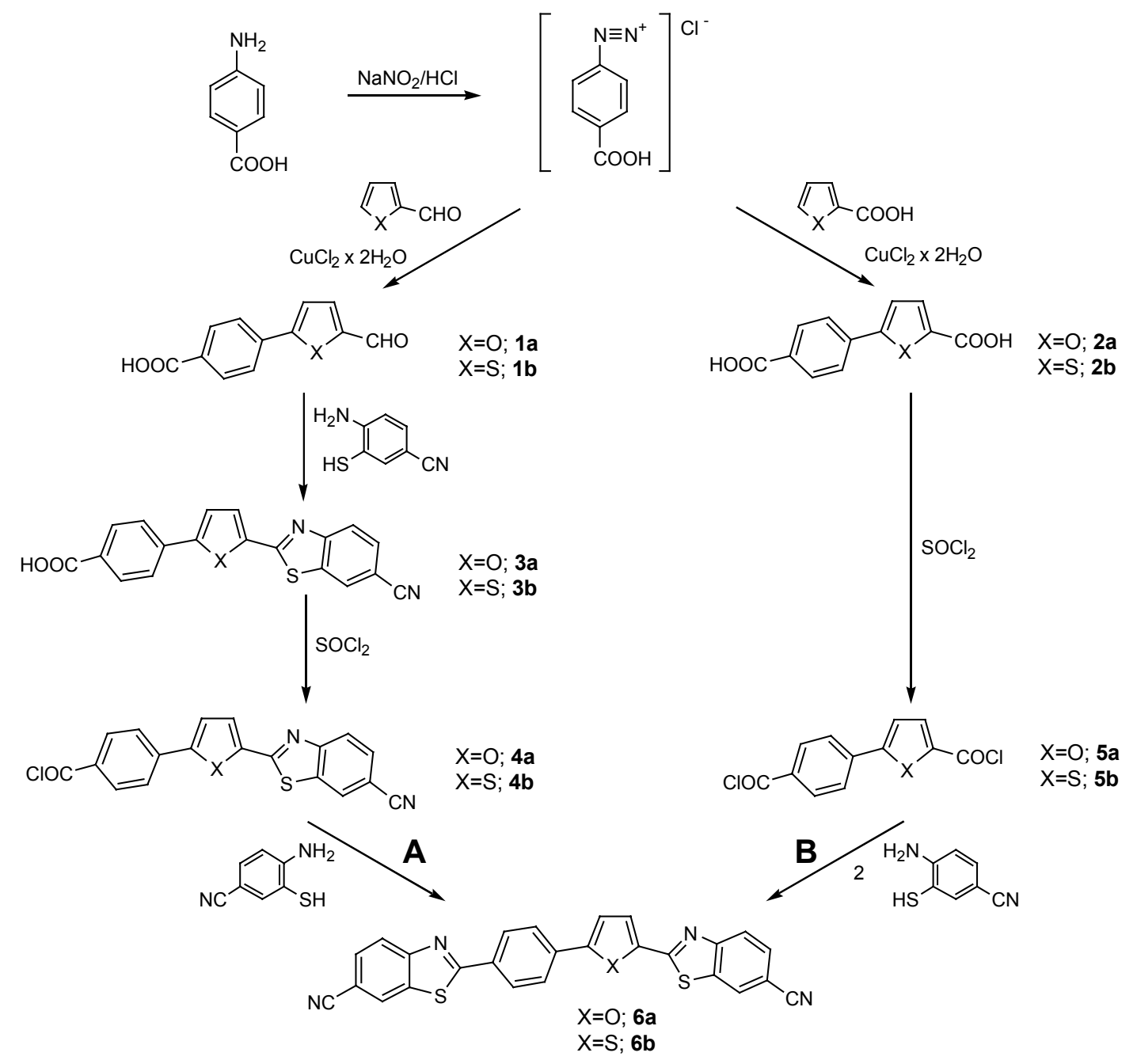

\section{Experimental}

\section{General}

Melting points were determined on a Koffler block apparatus and are uncorrected. IR spectra were determined with a Nicolet Magna 760 infrared spectrophotometer using KBr pellets. ${ }^{1} \mathrm{H}-\mathrm{NMR}$ spectral data were determined with a Brucker Avance DPX $300 \mathrm{MHz}$ NMR spectrometer with tetramethylsilane as an internal standard. Elemental analyses were carried out in the Microanalytical Laboratory at the Rugjer Boskovic Institute.

General Procedure for the Arylation of Compounds $\mathbf{1 a}, \mathbf{1 b}, \mathbf{2} \mathbf{a}$ and $\mathbf{2 b}$.

A solution of $p$-aminobenzoic acid $(75 \mathrm{mmol})$ in water $(120 \mathrm{~mL})$ and concentrated $\mathrm{HCl}(40 \mathrm{~mL})$ was cooled to $5^{\circ} \mathrm{C}$ and diazotized with a solution of $\mathrm{NaNO}_{2}(91 \mathrm{mmol})$ in $\mathrm{H}_{2} \mathrm{O}(35 \mathrm{~mL})$. After 20 min 
a solution of $75 \mathrm{mmol}$ of 2-furanaldehyde for 1a [20], 2-thiophenealdehyde for 1b [21], 2-furancarboxylic acid for $\mathbf{2 a}$ [20] or 2-thiophenecarboxylic acid for $\mathbf{2 b}$, respectively, in acetone (50 $\mathrm{mL})$ and a solution of $\mathrm{CuCl}_{2} \cdot 2 \mathrm{H}_{2} \mathrm{O}(23 \mathrm{mmol})$ in water $(25 \mathrm{~mL})$ were added to the stirred reaction mixture. The reaction mixture was left to stand at room temperature for two days with occasional shaking. After dilution with water $(500 \mathrm{~mL})$ the precipitated crystals were filtered off and washed with abundant hot water.

5-(4-carboxyphenyl)-2-thiophenecarboxylic acid (2b). Yield: 3.9 g (21\%); m.p.: >300 ${ }^{\circ} \mathrm{C}$ (from DMF water); IR (KBr): $1674(\mathrm{COOH}) \mathrm{cm}^{-1}$; ${ }^{1} \mathrm{H}-\mathrm{NMR}$ (300 MHz; DMSO-d $)_{6} \delta: 13.22$ (s, 2H, H-COOH), 8.01 (d, $J=8.4 \mathrm{~Hz}, 2 \mathrm{H}, \mathrm{H}$-arom.), 7.88 (d, $J=8.4 \mathrm{~Hz}, 2 \mathrm{H}, \mathrm{H}$-arom.), 7.76 (d, J=3.9, 1H, H-thioph.), 7.72 (d, J=3.9 Hz, 1H, H-thioph.); Anal. Calcd. for $\mathrm{C}_{12} \mathrm{H}_{8} \mathrm{O}_{4} \mathrm{~S}: \mathrm{C} 58.06, \mathrm{H}$ 3.25, S 12.92. Found: C 58.32, H 3.13, S 12.78 .

General Procedure for the Synthesis of Benzothiazolyl Compounds $\mathbf{3 a}$ and $\mathbf{3 b}$.

4-Amino-3-mercaptobenzonitrile $(21 \mathrm{mmol})$ was added to a solution of the appropiate aldehyde 1a or $1 \mathbf{b}(21 \mathrm{mmol})$ in pyridine $(60 \mathrm{~mL})$ and the stirred reaction mixture was refluxed $4 \mathrm{~h}$. The mixture was then poured into $2 \mathrm{M}$ hydrochloric acid $(400 \mathrm{~mL})$, and after cooling overnight, the crystalline product obtained was oxidized with an ethanolic solution of $\mathrm{FeCl}_{3}$ to obtain the benzothiazole compounds $\mathbf{3 a}$ and $\mathbf{3 b}$, respectively.

2-(4-Carboxyphenyl)-5-(6-cyanobenzothiazol-2-yl)furan (3a). Yield: 5.0 g (70\%); m.p.>300 ${ }^{\circ}$ (DMF); IR (KBr): $2210(\mathrm{CN}), 1670(\mathrm{COOH}) \mathrm{cm}^{-1} ;{ }^{1} \mathrm{H}-\mathrm{NMR}\left(300 \mathrm{MHz} ; \mathrm{DMSO}-\mathrm{d}_{6}\right) \delta: 13.18(\mathrm{~s}, 1 \mathrm{H}, \mathrm{H}-\mathrm{COOH})$, 8.78 (s, 1H, H-arom.), 8.50 (d, J=8.6 Hz, 1H, H-arom.), 8.07 (d, J=8.5 Hz, 2H, H-arom.), 8.01 (d, $J=8.5 \mathrm{~Hz}, 2 \mathrm{H}, \mathrm{H}$-arom.), 7.97 (d, $J=8.9 \mathrm{~Hz}, 1 \mathrm{H}, \mathrm{H}$-arom.), 7.68 (d, J=3.8 Hz, 1H, H-furan), 7.52 (d, $J=3.8 \mathrm{~Hz}, 1 \mathrm{H}, \mathrm{H}$-furan); Anal. Calcd. for $\mathrm{C}_{19} \mathrm{H}_{10} \mathrm{~N}_{2} \mathrm{O}_{3} \mathrm{~S}$ : C 65.89, H 2.91, N 8.09 S 9.26. Found: C 65.52, H 2.82, N 8.31 S 9.13.

2-(4-Carboxyphenyl)-5-(6-cyanobenzothiazol-2-yl)thiophene (3b). Yield: $4.5 \mathrm{~g}(62 \%)$; m.p. $>300^{\circ} \mathrm{C}$ (from DMF - ethanol); IR (KBr): $2224(\mathrm{CN}), 1687(\mathrm{COOH}) \mathrm{cm}^{-1} ;{ }^{1} \mathrm{H}-\mathrm{NMR}(300 \mathrm{MHz}$; TFA) $\delta: 8.33$ (s, 1H, H-arom.), 8.11 (d, J=4.2 Hz, 1H, H-thioph.), 8.06 (d, J=8.1 Hz, 2H, H-arom.), 7.99 (d, $J=8.7$ Hz, 1H, H-arom.), 7.91 (d, J=8.4 Hz, 1H, H-arom.), 7.68 (d, J=8.1 Hz, 2H, H-arom.), 7.56 (d, $J=4.2$ $\mathrm{Hz}, 1 \mathrm{H}$, H-thioph.); Anal. Calcd. for $\mathrm{C}_{19} \mathrm{H}_{10} \mathrm{~N}_{2} \mathrm{O}_{2} \mathrm{~S}_{2}$ : C 62.97, H 2.78, N 7.73 S 17.70. Found: C 62.95, H 2.82, N 8.01 S 17.58.

General Procedure for the Synthesis of Chlorocarbonyl Compounds $\mathbf{4 a}, \mathbf{4 b}, \mathbf{5 a}$ and $\mathbf{5 b}$.

Heating of a mixture of compounds $\mathbf{3 a}$ or $\mathbf{3 b}(11.5 \mathrm{mmol})$ with thionyl chloride $(230 \mathrm{mmol})$ or of compounds $\mathbf{2 a}$ or $\mathbf{2 b}(18 \mathrm{mmol})$ with thionyl chloride $(344 \mathrm{mmol})$ for $4 \mathrm{~h}$ on an oil bath at $85{ }^{\circ} \mathrm{C}$ 
afforded the corresponding monochlorocarbonyl compound $\mathbf{4 a}$ and $\mathbf{4 b}$ or the dichlorocarbonyl compound $\mathbf{5 a}$ and $\mathbf{5 b}$.

2-(4-Chlorocarbonylphenyl)-5-(6-cyanobenzothiazol-2-yl)furan (4a). Yield: $3.1 \mathrm{~g}(74 \%)$; m.p. $>300^{\circ} \mathrm{C}$ $\left(\mathrm{CHCl}_{3}\right)$; IR (KBr): $2210(\mathrm{CN}), 1760(\mathrm{COCl}) 1720(\mathrm{COCl}) \mathrm{cm}^{-1} ;{ }^{1} \mathrm{H}-\mathrm{NMR}(300 \mathrm{MHz}$; DMSO-d 6 ) $\delta$ : 8.78 (s, 1H, H-arom.), 8.20 (d, J=8.5 Hz, 1H, H-arom.), 8.07 (d, J=8.2 Hz, 2H, H-arom.), 8.00 (d, $J=8.5 \mathrm{~Hz}, 2 \mathrm{H}, \mathrm{H}$-arom.), 7.96 (d, $J=9.0 \mathrm{~Hz}, 1 \mathrm{H}, \mathrm{H}$-arom.), 7.68 (d, $J=3.8 \mathrm{~Hz}, 1 \mathrm{H}, \mathrm{H}$-furan), 7.51 (d, $J=3.8 \mathrm{~Hz}, 1 \mathrm{H}, \mathrm{H}$-furan).

2-(4-Chlorocarbonylphenyl)-5-(6-cyanobenzothiazol-2-yl)thiophene (4b). Yield: $2.7 \quad \mathrm{~g} \quad(62 \%)$; m.p.>300 ${ }^{\circ} \mathrm{C}\left(\mathrm{CHCl}_{3}\right)$;. IR (KBr): $2222(\mathrm{CN}), 1769(\mathrm{COCl}) 1720(\mathrm{COCl}) \mathrm{cm}^{-1}$; ${ }^{1} \mathrm{H}-\mathrm{NMR}(300 \mathrm{MHz}$; DMSO-d $_{6}$ ) $\delta: 8.75$ (s, 1H, H-arom.), 8.17 (d, J=8.7 Hz, 1H, H-arom.), 8.07 (d, J=3.9 Hz, 1H, Hthioph.), 8.03 (d, J=8.1 Hz, 2H, H-arom.), 7.95 (m, 3H, H-arom.), 7.86 (d, J=3.9 Hz, 1H, H-thioph.).

2-(4-Chlorocarbonylphenyl)-5-chlorocarbonylfuran (5a). Yield: $2.2 \mathrm{~g}(46 \%)$; m.p.102-106 ${ }^{\circ} \mathrm{C}$ (from benzene - cyclohexane); IR (KBr): $1740(\mathrm{COCl}) \mathrm{cm}^{-1} ;{ }^{1} \mathrm{H}-\mathrm{NMR}\left(300 \mathrm{MHz} ; \mathrm{CDCl}_{3}\right) \delta: 8.21(\mathrm{~d}, J=8.7$ Hz, 2H, H-arom.), 7.95 (d, $J=8.7 \mathrm{~Hz}, 2 \mathrm{H}, \mathrm{H}$-arom.), 7.60 (d, J=3.8 Hz, 1H, H-furan), 7.05 (d, J=3.8 $\mathrm{Hz}, 1 \mathrm{H}, \mathrm{H}$-furan).

2-(4-Chlorocarbonylphenyl)-5-chlorocarbonylthiophene (5b). Yield: $3.7 \mathrm{~g}(81 \%)$; m.p.88-92 ${ }^{\circ} \mathrm{C}$ (from benzene - cyclohexane); IR (KBr): $1771(\mathrm{COCl}) 1736(\mathrm{COCl}) \mathrm{cm}^{-1} ;{ }^{1} \mathrm{H}-\mathrm{NMR}\left(300 \mathrm{MHz} ; \mathrm{CDCl}_{3}\right) \delta$ : 8.20 (d, J=8.5 Hz, 2H, H-arom.), 7.99 (d, J=4.1 Hz, 1H, H-thioph.), 7.80 (d, J=8.5 Hz, 2H, H-arom.), 7.52 (d, $J=4.1 \mathrm{~Hz}, 1 \mathrm{H}, \mathrm{H}$-thioph.).

General Procedure for the Synthesis of bis-Benzothiazolyl Compounds $\mathbf{6 a}$ and $\mathbf{6 b}$.

A solution of monochlorocarbonyl compounds $4 \mathbf{a}$ or $\mathbf{4 b}(5.0 \mathrm{mmol}$, route A), or of dichlorocarbonyl compounds $\mathbf{5 a}$ or $\mathbf{5 b}(2.5 \mathrm{mmol}$, route B) in dry chlorobenzene $(100 \mathrm{~mL})$ was stirred under a stream of nitrogen. To these solutions 4-amino-3-mercaptobenzonitrile $(5.1 \mathrm{mmol})$ was added. The reaction mixture was heated under reflux under the stream of nitrogen for $70 \mathrm{~h}$. After cooling, a crystalline product was obtained.

2-[4-(6-Cyanobenzothiazol-2-yl)phenyl]-5-(6-cyanobenzothiazol-2-yl)furan (6a). Yields: $1.73 \mathrm{~g}(75 \%$, method A), 0.38 g (33\%, method B); m.p.>300 ${ }^{\circ} \mathrm{C}$ (DMF); IR (KBr): 2226 (CN) cm ${ }^{-1}$; ${ }^{1} \mathrm{H}-\mathrm{NMR}(300$ MHz; TFA) $\delta: 9.35$ (s, 1H, H-arom.), 9.27 (s, 1H, H-arom.), 9.03 (d, J=8.1 Hz, 2H, H-arom.), 8.968.80 (m, 6H, H-arom.), 8.74 (d, J=3.9 Hz, 1H, H-furan), 8.16 (d, J=3.9 Hz, 1H, H-furan). Anal. Calcd. for $\mathrm{C}_{26} \mathrm{H}_{12} \mathrm{~N}_{4} \mathrm{OS}_{2}$ : C 67.81, H 2.63, N 12.17 S 13.92. Found: C 67.86, H 2.54, N 12.10, S 14.02. 
2-[4-(6-Cyanobenzothiazol-2-yl)phenyl]-5-(6-cyanobenzothiazol-2-yl)thiophene (6b). Yields: $1.95 \mathrm{~g}$ (82\%, method A), 0.43 g (36\%, method B); m.p. $>300^{\circ} \mathrm{C}$ (DMF); IR (KBr): 2224 (CN) cm ${ }^{-1}$; ${ }^{1} \mathrm{H}-\mathrm{NMR}$

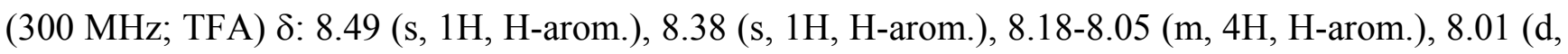
$J=3.9 \mathrm{~Hz}, 1 \mathrm{H}, \mathrm{H}$-thioph.), 7.97-7.92 (m, 4H, H-arom.), 7.70 (d, $J=4.0 \mathrm{~Hz}, 1 \mathrm{H}, \mathrm{H}$-thioph.); Anal. Calcd. for $\mathrm{C}_{26} \mathrm{H}_{12} \mathrm{~N}_{4} \mathrm{~S}_{3}$ : C 65.52, H 2.54, N 11.76, S 20.18. Found: C 65.38, H 2.51, N 11.66, S 20.32.

\section{References}

1. Lácová, M.; Chovancová, J.; Hýblová, O.; Varkonda, S. Synthesis and Pesticidal Activity of Acyl Derivatives of 4-chloro-2-aminobenzothiazole and the Products of their Reduction. Chem. Pap., 1991, 45, 411-418.

2. Chulák, I.; Sutorius, V.; Sekerka, V. Benzothiazole compound XXXV. Synthesis of 3-substituted 2-benzylbenzothiazolium Salts and their Growth-regulating Effect on Triticum Aestivum L. Chem. Pap. 1990, 44, 131-138.

3. Papenfuhs, T. Preparation of Benzothiazoles as Intermediates for Dyes, Plant Protectants and Pharmaceuticals. 1987, Ger. Offen. DE 3,528,032.

4. Bradshaw, T.D.; Bibby, M.C.; Double, J.A.; Fichtner, I.; Cooper, P.A.; Alley, M.C.; Donohue, S.; Stinson, S.F.; Tomaszewjski, J. E.; Sausville, E.A.; Stevens, M.F.G. Preclinical Evaluation of Amino Acid Prodrugs of Novel Antitumor 2-(4-amino-3-methylphenyl)benzothiazoles. Mol. Cancer Therapeutics. 2002, 1, 239-246.

5. Bradshaw, T.D.;Chua, M.S.; Browne, H.L.; Trapani, V.; Sausville, E.A.; Stevens, M.F.G. In Vitro Evaluation of Amino Acid Prodrugs of Novel Antitumor 2-(4-amino-3methylphenyl)benzothiazoles. Br. J. Cancer. 2002, 86, 1348-1354.

6. Hutchinson, I.; Jennings, S.A.; Vishnuvajjala. B.R.; Westwell, A.D.; Stevens, M.F.G. Antitumor Benzothiazoles. 16. Synthesis and Pharmacutical Properties of Antitumor 2-(4aminophenyl)benzothiazole Amino Acid Prodrugs. J. Med. Chem. 2002, 45, 744-747.

7. El-Sherbeny, M.A.; Synthesis of Certain Pyrimido[2,1-b]benzothiazole and Benzothiazolo[2,3b]quinazoline Derivatives for in Vitro Antitumor and Antiviral Activities. Arzeneim. Forsch. 2000, 50, 848-853.

8. Racanè, L.; Tralić-Kulenović, V.; Fišer-Jakić, L.; Boykin, D.W.; Karminski-Zamola, G. Synthesis of bis-Substituted Amidinobenzothiazoles as Potential Anti-HIV Agents. Heterocycles. 2001, 55, 2085-2098.

9. Sener, E.A.; Arpaci, O.T.; Yalcin, I.; Altanlar, N. Synthesis and Microbiological Activity of Some Novel 5-benzamido- and 5-phenylacetamido- Substituted 2-phenylbenzoxazole Derivatives. Farmaco. 2000, 55, 397-405.

10. Mruthyunjayaswamy, B.H.M.; Shanthaveerappa, B.K. Synthesis and Pharmacological Evaluation of 3,5-disubstituted indole-2-[N beta-(substituted benzopyran-2'-one-3'-carboxyl)]carboxy hydrazides and 2H-3-(various substituted indol-3'-yl)methyl-1,3-benzothiazoles. Indian J. Chem. Sect. B. 2000, 39, 433-439. 
11. Temiz-Arpaci, O.; Aki-Sener, E. Yalcin, I,; Altanlar, N. Synthesis and Antimicrobial Activity of Some 2-[p-substituted-phenyl]benzoxazol-5-yl-arylcarboxyamides. Arch. Pharm. Weinheim, Ger. 2002, 335, 283-288.

12. Delmas, F.; Di Giorgio, C.; Robin, M.; Azas, N.; Gasquet, M.; Detang, C.; Costa, M.; TimonDavid, P.; Galy, J.P. In Vitro Activities of Position 2 Substitution-bearing 6-nitro and 6-aminobenzothiazoles and their Corresponding Anthranilic Acid Derivatives Against Leishmania Infantum and Trichomonas Vaginalis. Antimicrob. Ag. Chemother. 2002, 46, 2588-2594.

13. Pattan, S.R.; Babu, S.N.N.; Angadi, J. Synthesis and Biological Activity of 2-amino [5'-(4'sulphonylbenzylidene)-2,4-thiazolidine dione]-7-(substituted)-6-fluoro benzothiazoles. Indian J. Heterocyclic Chem. 2002, 11, 333-334.

14. Mahmood-ul-Hasan; Chohan, Z.H.; Supuran, C.T. Antibacterial Zn(II) Compounds of Schiff Bases Derived from Some Benzothiazoles. Main Group Met. Chem. 2002, 25, 291-296.

15. Akyama, S.; Ochiai, T.; Nakatsuji, S.; Nakashima, K.; Ohkura, Y. Preparation and Evaluation of Fatty Acid Esters of Fluorescent $p$-Substituted Phenols as Substrates for Measurement of Lipase Activity. Chem. Pharm. Bull. 1987, 35, 3029-3032.

16. Farcasan, V.; Balazs, I. Derivati ai Furanului (VII). Stud. Univ. Babes-Bolyai-Ser. Chem. 1968, 13, 123-127.

17. Dotrong, M.; Mehta, R.; Balchin, G.A.; Tomlinson, R.C.; Sinsky, M.; Lee, C.Y.-C.; Evers, R.C. Synthesis, Processing, and Third-Order Nonlinear Optical Properties of Benzobisthiazole Polymers Containing Thiophene Moieties. J. Pol. Sci. Part A: Polym. Chem. 1993, 31, 723-729.

18. Racanè, L.; Tralić-Kulenović, V.; Karminski-Zamola, G.; Fišer-Jakić, L. Synthesis and Fluorescent Properties of Some New Unsymmetric bis-Benzothiazolyl Furans and Thiophenes. Monatsh. Chem. 1995, 126, 1375-1381.

19. Kornilov, M.Y.; Ruban, E.M.; Fredchuk, V.N., Starinskaya, E.V.; Buznik, M.V. Reaction of Chromophores in Asymetric 2,5-disubstituted Derivatives of Furan and Thiophene. Zh. Org. Khim. 1973, 9, 2577-2582.

20. Malinowski, S. Reaction of Diazo Compounds with Unsaturated Compounds II. Arylation of furfural. Polish J. Chem. 1953 27, 54-61.

21. Tralić-Kulenović, V.; Fišer-Jakić, L.; Lazarević, Z. Synthesis and Absorption Spectral Properties of Substituted Phenylfurylbenzothiazoles and their Vinylogues. Monatsh. Chem. 1994, 125, 209215.

22. Tralić-Kulenović, V.; Karminski-Zamola, G.; Racanè, L.; Fišer-Jakić, L. Synthesis of Symmetric and Unsymmetric bis-Cyanosubstituted Heterocycles. Heterocyclic Comm. 1998, 4, 423-428.

23. Bogert, M.T.; Naiman, B. Research on Thiazoles XX. J. Am. Chem. Soc. 1935, 57, 1529-1533.

Sample availability: Samples of compounds $\mathbf{2 b}, \mathbf{3 b}, \mathbf{6} \mathbf{a}$ and $\mathbf{6 b}$ are available from MDPI.

C 2003 by MDPI (http://www.mdpi.org). Reproduction is permitted for noncommercial purposes. 